\title{
The role of external beam radiotherapy in the treatment of papillary thyroid cancer
}

\author{
Nancy Lee ${ }^{1}$ and Michael Tuttle ${ }^{2}$ \\ ${ }^{1}$ Radiation Oncology and ${ }^{2}$ Endocrine Service, Memorial Sloan-Kettering Cancer Center, New York, NY, USA
}

\begin{abstract}
The role of external beam radiotherapy (EBRT) in treating thyroid cancer has brought forth controversy. Due to various histologic presentations and different natural histories, there is no uniform approach/recommendation among centers and/or authorities regarding the role of EBRT for thyroid cancer. This is particularly true for papillary thyroid carcinoma (PTC) where the clinical course can range from a disease that is cured with simple surgery to an aggressive form of poorly differentiated thyroid cancer with high rates of recurrence/death from disease. In addition, because the majority of the patients with PTC undergo postoperative radioactive iodine (RAI) treatment, the question remains as to what is the exact role of EBRT for PTC in the setting of RAI treatment?

In this issue of Endocrine-Related Cancer, Chow and colleagues identified indications for EBRT and RAI therapy for PTC based on a retrospective review of 1300 patients. The authors concluded that postoperative RAI treatment is indicated in patients with pT2-pT4, pN0-pN1b while postoperative EBRT is recommended for patients with gross residual, positive margin, pT4, $\mathrm{pN} 1 \mathrm{~b}$, and lymph nodes $>2 \mathrm{~cm}$ disease. Other centers have also published their experience on the value of EBRT for PTC but with different indications. The reasons for the variations from different centers are complex. However, when all published results are taken together, the findings confirm the added value of EBRT to the present management of PTC in a select group of patients, particularly those with high risk features. In this commentary, these issues will be discussed and recommendations regarding the role of EBRT will be given.
\end{abstract}

Endocrine-Related Cancer (2006) 13 971-977

\section{Overview}

External beam radiotherapy (EBRT) for papillary thyroid cancer (PTC) has been a controversial topic in the field of endocrinology, head and neck surgery, as well as radiation oncology. There is no consensus on who is a candidate for EBRT as each center has its own philosophy in terms of the role of EBRT for PTC. For example, one center reports the importance of prophylactic EBRT in differentiated thyroid cancer even among patients with no microscopic residual disease based on improvement in the locoregional relapse-free and cause-specific survival in patients who underwent adjuvant EBRT after complete surgical resection (Esik et al. 1994). In contrast, investigators from other centers do not routinely recommend EBRT for PTC as no improvement in overall survival was observed (Benker et al. 1990, Lin et al. 1997). In fact, there was a higher survival rate among those who did not receive EBRT in one study (Lin et al. 1997). In addition, Mazzaferri et al. showed the routine use of
EBRT to have an adverse effect on outcome, but this observation was likely to be secondary to the marked selection bias of very high risk patients selected for EBRT in this series (Mazzaferri \& Young 1981).

The age of the patient also has been factored into the decision making for EBRT in many centers. For example, it is generally accepted that no postoperative EBRT is necessary for a young patient with limited gross residual disease where there is good demonstration of RAI uptake (O'Connell et al. 1994, Tsang et al. 1998, Brierley et al. 2005). Others, however, failed to find any correlation of outcome with age (Benker et al. 1990). Due to these opposing views from different groups, confusion arises for both the patient as well as the physician, as EBRT might be strongly recommended in one center but not recommended at all at another center. The discrepancies in findings between centers are largely due to the retrospective nature of the studies with variation in patient selection, 
EBRT technique, EBRT dosing, as well as irradiated volume. Our colleagues from Germany attempted to answer this controversial topic by conducting a randomized controlled clinical trial that involved 44 different centers in Germany, Austria and Switzerland. The trial aimed to test the role of EBRT as adjuvant treatment for differentiated PTC (Biermann et al. 2003). However, due to the limited acceptance of EBRT among these centers, the trial stopped prematurely and did not meet the targeted patient accrual.

In this issue of Endocrine-Related Cancer, we read another retrospective review on the role of EBRT for PTC from Hong Kong. Chow and colleagues reported their experience of close to 1300 patients where 817 patients underwent RAI therapy alone, 28 patients received EBRT alone, and 163 patients underwent combined treatments with RAI and EBRT after appropriate surgical resection. The median follow-up was 10 years. The authors concluded that EBRT is indicated after surgical re-section in patients with gross residual disease, positive resection margins, pT4, $\mathrm{pN} 1 \mathrm{~b}$, or a lymph node size of $>2 \mathrm{~cm}$ (Chow et al. 2006). This paper certainly is a valuable addition to the currently available literature regarding this topic; however, as stated earlier, variations in patient selection can confound the results. Before routinely recommending EBRT for PTC, one must always keep in mind the associated morbidity of EBRT for thyroid cancer and weigh that against any benefit, particularly if the benefit is only in locoregional control of the disease without prolonging disease specific survival. Therefore, it is the goal of this editorial to place the data from Chow et al. (2006) in proper context and taking into consideration other reported experiences.

\section{External beam radiotherapy for gross residual disease}

Studies have shown that the locoregional control rates for patients who have gross residual disease after surgical resection can be improved with EBRT (Sheline et al. 1966). Tubiana et al. report their experience of 97 patients who received EBRT in which the surgeons considered tumor re-section to be incomplete leaving macroscopic residual disease (Tubiana et al. 1985). At 15 years, the authors report a $39 \%$ relapse-free and $57 \%$ overall survival rate among patients who received postoperative EBRT. The same authors also report their experience with 17 patients with inoperable disease treated with EBRT alone. At 5 years, the relapse-free and overall survival rates were $55 \%$ and $60 \%$, respectively. O'Connell et al. reported on 49 patients treated with EBRT who had gross residual disease (O'Connell et al.
1994). A complete response was seen in $41 \%$ of patients with PTC while $22 \%$ achieved partial response with an overall survival rate of $27 \%$. Lastly, Tsang et al reported on the effects of EBRT in 33 patients with gross residual disease, 13 of whom also had RAI therapy. The 5-year cause-specific survival and local relapse-free rates were $65 \%$ and $62 \%$, respectively (Tsang et al. 1998).

In the retrospective review published in this issue, $17 \%$ of the patients were classified as having gross residual disease. Among these 217 patients, 23\% had RAI therapy alone, $7.4 \%$ had EBRT and 52\% had both RAI and EBRT. Approximately $18 \%$ of the patients did not have any radiation therapy. The authors found that EBRT in patients with gross residual disease not only improved locoregional control but also improved the cause-specific survival when compared to those who had not received EBRT. The locoregional recurrence-free survival increased from $24 \%$ to $63 \%$ at 10 years while the cause-specific survival improved from $50 \%$ to $74 \%$. The authors further classified patients into those with palpable disease, which consisted of 58 patients, versus those with nonpalpable disease $(n=137)$. In patients with palpable disease, EBRT significantly improved the 2-year locoregional control from $6 \%$ to $23 \%(P=0.03)$. In addition, patients with non-palpable gross locoregional disease also had an increase of 10-year locoregional control from $39 \%$ to $80 \% \quad(P<0.0001)$ with the addition of EBRT.

These published series confirmed the benefit of EBRT for gross residual disease after thyroidectomy. Therefore, it is the authors' opinion that in addition to RAI therapy, EBRT should be considered as an integral part of the management for select patients with gross disease after surgical resection. This appears to be particularly important in older patients who often have more poorly differentiated thyroid cancer that is likely to be more aggressive and less likely to concentrate tumoricidal doses of RAI. With current available radiotherapy techniques such as intensity-modulated radiation therapy (IMRT; see below), a higher dose of radiation such as 70 Gy can be safely given to the gross residual disease without exceeding the tolerance of surrounding critical structures. Therefore, a therapeutic dose for gross disease can be achieved. However, it is also the authors' opinion that not everyone requires postoperative EBRT, even in the presence of gross residual disease. For example, postoperative EBRT is probably not necessary for a young patient who presents with limited gross residual disease where the disease demonstrates good $\mathrm{I}^{131}$ uptake, in which tumoricidal doses are likely to be achieved with RAI alone (Brierley \& Tsang 1999, Mazzarotto et al. 2000). 


\section{External beam radiotherapy for micro- scopic residual disease (extrathyroidal extension, pT4, positive margin, invasion of the major structures, and/or multiple positive lymph nodes)}

Completeness of surgical resection is occasionally not achievable due to the extent of disease on presentation. No microscopic residual disease is defined when the surgeon removes all gross disease along with a good margin of uninvolved tissue (Shaha 2004, Patel et al. 2005) Most patients will have microscopic residual disease if the tumor has been shaved of the trachea, larynx or esophagus via blunt dissection. Additionally, even though the surgeon feels that all evidence of gross disease has been removed, minor microscopic extrathyroidal extension can be sometimes identified on histological specimens that were not recognized intraoperatively. O'Connell et al. reported a very good local control of $81 \%$ even in the presence of microscopic residual disease when EBRT is added as part of the management (O'Connell et al. 1994). Overall, the 5-, 10 - and 15-year overall survival rates were $85 \%, 60 \%$ and $15 \%$ respectively. Similarly, Sheline et al. reported their experience of 12 patients with microscopic residual disease where 11 remained alive and well with no evidence of recurrence (Sheline et al. 1966). However, these small studies are difficult to put into context because many patients with microscopic extrathyroidal extension respond very well to RAI, making the addition of EBRT in this setting questionable in well-differentiated tumors.

However, gross extrathyroidal extension has been recognized as a poor prognostic feature in differentiated thyroid cancer (Tsang et al. 1998, Patel et al. 2005). A retrospective review of patients treated at the MD Anderson Cancer Center showed that the risk of local recurrence was higher in patients with more extensive local tumor (Samaan et al. 1992). When the disease was confined to the thyroid gland, the local recurrence rate was $19 \%$ but this increases to $36 \%$ when there is infiltration of the soft tissues. When there is microscopic residual disease, multiple studies support the use of adjuvant radiotherapy after total thyroidectomy (Philips et al. 1993, O'Connell et al. 1994, Farahati et al. 1996, Kim et al. 2003, Keum et al. 2006, Meadows et al. 2006). Most of these series report a significant improvement in locoregional control in patients who present with pT4 and/or with positive lymph node involvement. Several series also noted that older patients ( $>40$ years of age) with multiple positive lymph nodes or extrathyroidal extension had better locoregional control of their disease with the addition of EBRT. Chow and colleagues also found that EBRT can improve the local recurrence-free survival in patients with either pT4 disease, multiple positive lymph nodes, or with any lymph node $>2 \mathrm{~cm}$. EBRT has also been shown to be effective particularly in patients who had disease invading the trachea resulting in either macroscopic or microscopic residual disease (Keum et al. 2006). The 10-year local progression-free survival rate was significantly better than unirradiated controls, $81 \%$ versus $38 \%, P<0.01$.

Given the above data, in the light of no prospective randomized trials available, EBRT should be considered in patients who are at high risk for locoregional recurrence. High risk features include microscopic residual disease involving surrounding major structures that is likely to remain even after meticulous surgical removal of all gross disease, especially in older patients, and if the histology of the tumor suggests that it is unlikely to respond to therapeutic RAI (Cooper et al. 2006). More data is needed to determine if EBRT is beneficial to patients in whom the only evidence of extrathyroidal extension is minor invasion through the thyroid capsule into surrounding perithyroidal fat that has been completely resected with the thyroidectomy specimen.

EBRT should also be considered in elderly patients with multiple positive lymph nodes. Without additional data showing a survival benefit, we do not currently advocate EBRT for the sole indication of cervical lymph node involvement in either the young or the elderly. While these patients are at significant risk of recurrence, these recurrences can usually be controlled with additional surgery or RAI without an apparent major change in overall survival.

Before routinely recommending EBRT for all patients with high risk features, one must factor the toxicities associated with EBRT into the equation. Therefore, a multidisciplinary approach is recommended in the management of PTC due to the complex nature of these patients at presentation. In some patients, the significant morbidities associated with EBRT such as mucositis, pharyngitis and hoarseness may outweigh the potential benefit in terms of local control without an overall survival benefit (Tuttle et al. 2004).

\section{External beam radiotherapy for recurrent PTC}

The standard treatment for patients with PTC who relapse in the neck is surgery often followed by additional RAI and continued TSH suppression treatment. A neck dissection is often performed in patients with nodal recurrence (Brierley \& Tsang 1999, 
Shaha 2004). RAI treatment has also been used to treat nodal recurrences. Therefore, the question is when to consider adjuvant EBRT. Many centers advocate EBRT when there is infiltration of the soft tissues of the neck or extracapsular spread of the lymph nodes at the time of recurrence. One series reported on five patients who received EBRT at the time of locoregional recurrence and no further relapses were seen (Sheline et al. 1966). At another center, $50 \%$ of the patients no longer experienced a relapse regionally when EBRT was added to surgery (VassilopoulouSelin et al. 1996). Finally, in situations were there is extensive extrathyroidal extension, the addition of EBRT at the time of recurrence should be considered (Brierley \& Tsang 1999).

Due to the morbidity associated with EBRT, some centers recommend reserving EBRT and use salvage surgery and/or RAI therapy at the time of recurrence (Shaha 2004, Kepal et al. 2005). Most recurrences can be effectively treated with additional surgery and RAI, without the addition of routine EBRT. In the recent management guideline by the American Thyroid Association Guidelines Taskforce, the use of EBRT should be considered for patients with gross residual tumor in whom further surgery or RAI treatment are likely to be ineffective (Cooper et al. 2006). Lastly, whether EBRT can reduce the risk of additional locoregional recurrences or increase the relapse-free rates when patients present with recurrent disease remains to be seen.

\section{Radiotherapy dose for PTC}

A wide spectrum of EBRT doses has been used in the treatment of PTC, ranging from $30 \mathrm{~Gy}$ in the older series to 70 Gy more recently (Sheline et al. 1966, Rosenbluth et al. 2005). Studies included patients with either gross or microscopic residual disease, which confounds the interpretation of the findings. However, radiation oncologists concur that the lower doses are probably suboptimal in the modern era (Brierley \& Tsang 1999). Lower doses were used historically because older radiotherapy methods could not deliver an adequate dose of radiation to the thyroid bed without exceeding the tolerance of the posteriorly located spinal cord (see technique section below). Despite these limitations, one study reported a 50\% (8 out of 15) control rate of PTC patients who underwent EBRT with gross residual or inoperable disease (Sheline et al. 1966). Follow-up ranged from 1 to 25 years. In the more modern series, the recommended doses for gross residual or inoperable disease typically range from $60 \mathrm{~Gy}$ to $70 \mathrm{~Gy}$ with the latter used preferentially for inoperable disease
(Brierley et al. 2005, Rosenbluth et al. 2005, Meadows et al. 2006). O'Connell et al. reported the Royal Marsden experience where the 5-year overall survival rate was $27 \%$ for patients with gross residual disease (O'Connell et al. 1994). The authors emphasized the importance of achieving a radical radiotherapy dose which is required to eradicate gross disease, as PTC is relatively radioresistant with a slow regression rate. Doses for microscopic residual disease range from 50 Gy to $70 \mathrm{~Gy}$ in reported series, with a median of $60 \mathrm{~Gy}$. A median dose of $60 \mathrm{~Gy}$ was also used in the study by Chow and colleagues. Interestingly, Ford and colleagues noted that local recurrence and overall survival are related to the dose prescribed (Ford et al. 2003). Although no firm conclusions can be drawn from their retrospective review of 41 patients who underwent EBRT after surgery, based on the observed doseresponse relationship in this small cohort of patients, a total dose of at least $50 \mathrm{~Gy}$ and possible higher is needed to impact local control and possibly survival.

\section{Radiotherapy volume for PTC}

What volume of tissue should be included when patients undergo EBRT? Differences among radiation oncologists are apparent as the irradiated volume for PTC varies tremendously between centers. Radiation can be limited to the thyroid bed only or also include the bilateral neck compartments (O'Connell et al. 1994, Tsang et al. 1998, Kim et al. 2003, Brierley et al. 2005, Rosenbluth et al. 2005, Meadows et al. 2006, Keum et al. 2006). There are also different opinions as to the extent of the neck that needs to be irradiated. Some define the superior border starting from the tip of the mastoid process while others define the neck at risk starting from the upper level of the thyroid bed. Most agree that the inferior extent of the disease should include the upper mediastinum, although some advocate the supraclavicular fossae as the inferior border. Chow and colleagues note that because of the close proximity of the thyroid bed to the regional lymph nodes, the target volume covered both sides of the neck from the jaw to the angle of Louis. Bilateral supraclavicular fossae and the sternal notch were also included. These large differences can partially explain the differences in the observed locoregional control of disease and particularly the treatment-related toxicities (Schuck et al. 2003). The acute toxicities reported include mucositis, pharyngitis, dysphagia, hoarseness and radiation dermatitis, while late complications include skin fibrosis, tracheal stenosis and esophageal stricture. A uniform guideline for target delineation is needed. Until then, radiation oncologists should consider the toxicities 
associated with treatment and only include the area(s) at highest risk for recurrence in the target volume.

\section{New advances in external beam radiotherapy technology}

Due to the close proximity of the thyroid gland to the spinal cord and the lung, adequate doses of EBRT cannot be delivered without exceeding the tolerance of these critical organs when using conventional twodimensional (2D) radiotherapy techniques (Posner et al. 2000, Nutting et al. 2001). For example, because the tolerance of the posteriorly located spinal cord to the thyroid bed is $45 \mathrm{~Gy}$, it is very difficult to deliver $60 \mathrm{~Gy}$ to control microscopic residual disease. To overcome this limitation, several dosimetric studies have been carried out recently on the use of IMRT in the treatment of thyroid cancer. They have uniformly shown improvements in target coverage while reducing the spinal cord dose. Nutting et al. compared IMRT with three-dimensional conformal radiotherapy (3D-CRT) and conventional 2D techniques. The authors reported that although 3D-CRT reduced normal tissue irradiation compared to the older 2D conventional techniques, 3D-CRT did not improve target volume coverage nor did it limit the spinal cord to below $45 \mathrm{~Gy}$. Furthermore, the authors noted that only IMRT improved the target volume coverage along with preservation of the spinal cord (Nutting et al. 2001). Another study from the University of California-San Francisco compared conventional 2D techniques with IMRT (Posner et al. 2000). The authors concluded that using intensity-modulation, they were able to achieve the therapeutic dose while respecting the tolerance to the spinal cord. Furthermore, IMRT has the ability to give differential dosing to different parts of the postoperative bed. In the same IMRT plan, gross disease can receive a higher total dose as well as dose per fraction when compared to areas with possible microscopic residual disease. This is also known as dose-painting IMRT, where different regions within the irradiated volume receive different dosing while respecting the tolerance of the surrounding normal tissues. An example of a patient undergoing postoperative EBRT can be seen in Fig. 1. Notice that the target volume received $60 \mathrm{~Gy}$ while the spinal cord received less than $45 \mathrm{~Gy}$.

In addition to the dosimetric reports on the advantage of IMRT in the treatment of thyroid cancer, one series was able to give a median total dose of $65 \mathrm{~Gy}$ by using IMRT, which is a 20 Gy increase in dose compared to older radiotherapy techniques (Meadows et al. 2006). Another preliminary report by Rosenbluth et al. looked at

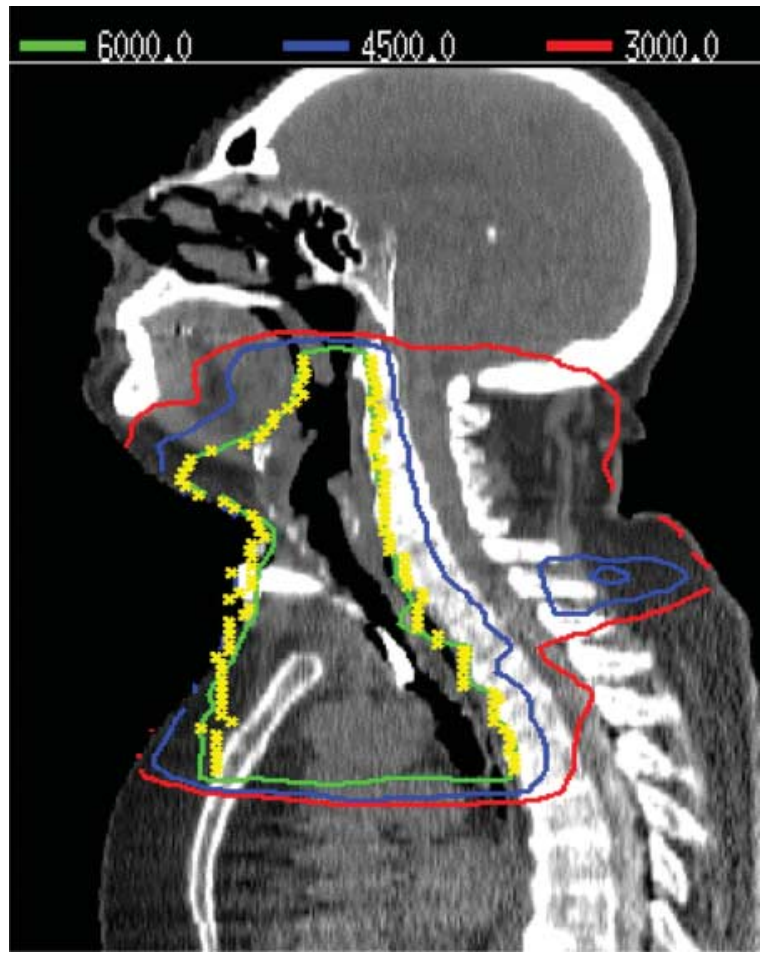

Figure $1 \mathrm{~A}$ patient undergoing postoperative EBRT. Target volume is in yellow; green isodose line represents $60 \mathrm{~Gy}$; blue isodose line represents $45 \mathrm{~Gy}$. Notice that the spinal cord is outside of the $45 \mathrm{~Gy}$ isodose line.

20 non-anaplastic thyroid cancer patients from 7/01 to 1/04 treated with IMRT at Memorial Sloan-Kettering Cancer Center (Rosenbluth et al. 2005). Most of these patients had recurrent disease or T4N1 disease. Two local failures and six deaths have been noted resulting in a 2-year local progression-free rate of $85 \%$ and overall survival rate of $60 \%$. Acute mucositis, pharyngitis, laryngeal and skin toxicities were experienced by seven, three, three and two patients, respectively. The authors found IMRT to be feasible and well-tolerated, although longer follow-up is needed to assess the full extent of toxicity and outcome.

\section{Summary}

The role of EBRT in the treatment of PTC is controversial. Unfortunately, no randomized studies have been performed to date to evaluate EBRT after surgical resection. Different patient selection, EBRT techniques, EBRT doses and EBRT irradiated volumes confound the interpretation of the available data. In summarizing the data from previous published series along with the review in this current issue (Chow et al. 2006), it is the authors' opinion that routine 
postoperative EBRT should not be used for all PTC patients. EBRT should only be considered in a highly select group of patients with (1) gross residual disease, (2) older patients with microscopic residual disease remaining after meticulous resection of tumor invading surrounding structures who are at high risk for recurrence and unlikely to respond to RAI therapy, and (3) patients with recurrent disease that cannot be adequately controlled with additional surgery and RAI. While this editorial has focused on the role of EBRT in the neck, it is clear that EBRT also can play a major role in the therapy of bone and brain metastases as well, but these issues are beyond the scope of this editorial.

Unfortunately, a major limitation of all published series is that none of the recommendations were based on risk group stratifications (Shaha 2004). Future studies should first stratify patients into low, intermediate and high risk groups, and subsequently recommend EBRT based on the patterns of treatment failure in the different risk groups (Shaha et al. 1998). In addition, because the response of the tumor to RAI is a major factor in prognosis in patients with microscopic residual disease, careful analysis of the histology, RAI scan results, and 2-[18F]fluoro-2deoxy-D-glucose-position emission tomography scan results are required to properly select those patients most likely to benefit from EBRT. Until then, the use of EBRT should be highly selected and the benefits should be weighed against the toxicities associated with treatment.

\section{References}

Benker G, Olbricht T, Reinwein D, Reiners C, Sauerwein W, Krause U, Mlynek ML \& Hirche H 1990 Survival rates in patients with differentiated thyroid carcinoma. Influence of postoperative external radiotherapy. Cancer 65 1517-1520.

Biermann M, Pixberg MK, Schuck A, Heinecke A, Kopcke W, Schmid KW, Dralle H, Willich N \& Schober O 2003 Multicenter study differentiated thyroid carcinoma (MSDS). Diminished acceptance of adjuvant external beam radiotherapy. Nuclearmedizin 42 244-250.

Brierley JD \& Tsang R 1999 External-beam radiation therapy in the treatment of differentiated thyroid cancer. Seminars in Surgical Oncology 16 42-49.

Brierley J, Tsang R, Panzarella T \& Bana N 2005 Prognostic factors and the effect of treatment with radioactive iodine and external beam radiation on patients with differentiated thyroid cancer seen at a single institution over 40 years. Clinical Endocrinology 63 418-427.

Chow SM, Yau S, Kwan CK, Poon PCM \& Law SCK 2006 Local and regional control in patients with papillary thyroid carcinoma: specific indications of external radiotherapy and radioactive iodine according to $\mathrm{T}$ and $\mathrm{N}$ categories in AJCC 6th edn. Endocrine-Related Cancer 13 1161-1174.

Cooper DS, Doherty GM, Haugen BR, Kloos RT, Lee SL, Mandel SJ, Mazzaferri EL, Melver B \& Tuttle RM 2006 Management guidelines for patients with tryoid nodules and differentiated thyroid cancer. Thyroid 16 1-33.

Esik O, Nemeth G \& Eller J 1994 Prophylactic external irradiation in differentiated thyroid cancer: a retrospective study over a 30-year observation period. Oncology 51 372-379.

Farahati J, Reiners C, Stuschke M, Muller SP, Stuben G, Sauerwein W \& Sack H 1996 Differentated thyroid cancer. Impact of adjuvant external beam radiotherapy in patients with perithryoidal tumor infiltration (stage pT4). Cancer 77 172-180.

Ford D, Giridharan S, McConkey C, Hartley A, Brammer C, Watkinson JC \& Glaholm J 2003 External beam radiotherapy in the management of differentiated thyroid cancer. Clinical Oncology 15 337-341.

Keum KC, Suh YG, Koom WS, Cho JH, Shim SJ, Lee CG, Park CS, Chung WY \& Kim GE 2006 The role of postoperative external-beam radiotherapy in the management of patients with papillary thyroid cancer invading the trachea. International Journal of Radiation Oncology, Biology, Physics 65 474-480.

Kim TH, Yang DS, Jung KY, Kim CY \& Choi MS 2003 Value of external irradiation for locally advanced papillary thyroid cancer. International Journal of Radiation Oncology, Biology, Physics 55 1006-1012.

Lin JD, Tsang NM, Huang MJ \& Weng HF 1997 Results of external beam radiotherapy in patients with well differentiated thyroid carcinoma. Japanese Journal of Clinical Oncology 27 244-247.

Mazzaferri EL \& Young RL 1981 Papillary thyroid carcinoma: a 10 year follow-up report of the impact of therapy in 576 patients. American Journal of Medicine $\mathbf{7 0}$ 511-518.

Mazzarotto R, Lora CO \& Casara RD 2000 The role of external beam radiotherapy in the management of differentiated thyroid cancer. Biomedicine and Pharmocotherapy 54 345-349.

Meadows KM, Amdur RJ, Morris CG, Villarer DB, Mazzaferri EL \& Mendenhall WM 2006 External beam radiotherapy for differentiated thyroid cancer. American Journal of Otolaryngology 27 24-28.

Nutting CM, Convery DJ, Cosgrove VP, Rowbottom C, Vini L, Harmer C, Dearnaley DP \& Webb S 2001 Improvements in target coverage and reduced spinal cord irradiation using intensity-modulated radiotherapy (IMRT) in patients with carcinoma of the thyroid gland. Radiotherapy and Oncology 60 173-180.

O'Connell MEA, A'hern RP \& Harmer CL 1994 Results of external beam radiotherapy in differentiated thyroid carcinoma: a retrospective study from the Royal Marsden Hospital. European Journal of Cancer 30A 733-739. 
Patel KN \& Shaha AR 2005 Locally advanced thyroid cancer. Current Opinion in Otolaryngology \& Head and Neck Surgery 13 112-116.

Philips P, Hanzen C, Andry G, Houtte V \& Fruuling J 1993 Postoperative irradiation for thyroid cancer. European Journal of Surgical Oncology 19 399-404.

Posner MD, Quivey JM, Akazawa PF, Xia P, Akazawa C \& Verhey LJ 2000 Dose optimization for the treatment of anaplastic thyroid carcinoma: a comparison of treatment planning techniques. International Journal of Radiation Oncology, Biology, Physics 48 475-483.

Rosenbluth BD, Serrano V, Happersett L, Shaha AR, Tuttle RM, Narayana A, Wolden SL, Rosenzweig KE, Chong LM \& Lee NY 2005 Intensity-modulated radiation therapy for the treatment of nonanaplastic thyroid cancer. International Journal of Radiation Oncology, Biology, Physics 63 1419-1426.

Samaan NA, Schultz PN \& Kickey RC 1992 The results of various modalities of treatment of well differentiated thryoid carcinomas: a retropsective review of 1599 patients. Journal of Clinical Endocrinology and Metabolism 75 714-720.

Schuck A, Biermann M, Pixberg MK, Muller SB, Heinecke A, Schober O \& Willich N 2003 Acute toxicity of adjuvant radiotherapy in locally advanced differentiated thyroid carcinoma. Strahlentherapie und Onkologie 173 832-839.
Shaha AR 2004 Implications of prognostic factors and risk groups in the management of differentiated thyroid cancer. Laryngoscope 114 393-402.

Shaha AR, Shah JP \& Loree TR 1998 Patterns of failure in differentiated carcinoma of the thyroid based on risk groups. Head and Neck 20 26-30.

Sheline GE, Galante M \& Lindsay S 1966 Radiation therapy in the control of persistent thryoid cancer. American Journal of Roentgenology, Radium Therapy, and Nuclear Medicine 97 923-930.

Tsang RW, Brierley JD, Simpson WJ, Panzarella T, Gospodarowicz MK \& Sutcliffe SB 1998 The effects of surgery, radioiodine, and external radiation therapy on the clinical outcome of patients with differentiated thyroid carcinoma. Cancer 82 375-388.

Tubiana M, Haddad E, Schlumberger M, Rougier P, Laplanche A, Benhamou E \& Gardet P 1985 External radiotherapy in thyroid cancers. Cancer 55 2062-2071.

Tuttle M, Robbins R, Larson SM \& Strauss HW 2004 Challenging cases in thyroid cancer: a multidisciplinary approach. European Journal of Nuclear Medicine and Molecular Imaging 31 605-612.

Vassilopoulou-Selin R, Schultz PN \& Haynie TP 1996 Clinical outcome of patients with papillary thyroid carcinoma who have recurrence after initial radioactive iodine therapy. Cancer 78 493-501. 